\title{
BATERÍAS QUE ESTÁN CAMBIANDO el mundo. Premio Nobel DE Química 2019
}

\author{
Batteries that are Changing the world. 2019 Nobel Prize in \\ Chemistry
}

\section{Daniel Guerra Giráldez ${ }^{1}$}

\section{RESUMEN}

El Premio Nobel de Química 2019 fue otorgado a John B. Goodenough, M. Stanley Whittingham y Akira Yoshino por sus aportes al desarrollo de las baterías de litio. Con sus investigaciones, ellos han creado las condiciones para una sociedad futura inalámbrica y libre de combustible fósil. Así, se producirán grandes cambios en lo social, ambiental, económico y geopolítico cuando las baterías de litio alimenten la mayoría de los automóviles, fábricas y casas.

Palabras claves: Premio Nobel, química, batería, litio.

\section{ABSTRACT}

The 2019 Nobel Prize in Chemistry was awarded to John Goodenough, M. Stanley Whittingham and Akira Yoshino by the contributions to the development of lithium-ion batteries. Through their researches, they have created the conditions for a wireless and fossil fuelfree future society. So, there will be major social, environmental, economic and geopolitical changes when lithium batteries feed most cars, factories and homes.

Keywords: Nobel Prize, chemistry, battery, lithium.

1 Premio Nobel de Química 2019 fue otorgado a John B. Goodenough, M. Stanley Whittingham y Akira Yoshino por sus aportes al desarrollo de las baterías de litio. Décadas de descubrimientos y acumulación de mejoras ya revolucionaron los dispositivos móviles. Ahora, estas nuevas baterías comienzan a alimentar automóviles y distritos enteros.

1 Doctor en Ciencias Biomédicas. Laboratorio de Moléculas Individuales, Facultad de Ciencias y Filosofía ACT, UPCH.
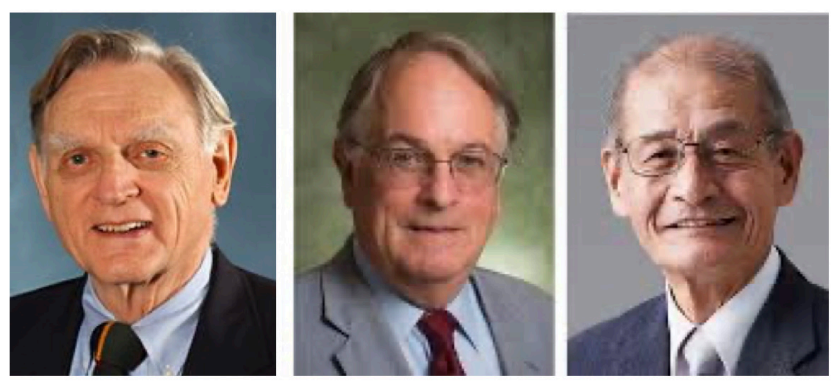

De izquierda a derecha: John B. Goodenough, M. Stanley Whittingham y Akira Yoshino

Todos los aspectos de nuestra forma de vivir giran en torno a un uso cada vez mayor de energía. Las fuentes renovables como la hidráulica, solar o eólica pueden producir una alta potencia pero, por lo general, esta debe ser utilizada inmediatamente o se pierde. Es difícil imaginarlo, pero al encender una luz en la ciudad, esa energía fue generada hace pocos segundos por una turbina en una caída de agua lejos de allí. En cambio, un litro de gasolina puede entregar $9 \mathrm{kWh}$ hoy, mañana o dentro de unos años, sin perder en nada su capacidad. En todo el mundo reconocemos los problemas ambientales relacionados con los combustibles fósiles pero, para abandonarlos y que las energías renovables puedan cubrir nuestra demanda, estas no solo deben entregar la cantidad de energía suficiente sino que además deben acumularse y transportarse 
fácilmente. Almacenar energía en un volumen reducido, de manera estable y con alta potencia es, por ende, un dilema clave para la economía y la esperanza de mitigar el cambio climático global. Veremos, no obstante, que sigue siendo muy difícil vencer las virtudes de los compactos y durables combustibles fósiles. Todos los que usamos dispositivos portátiles hemos notado el vertiginoso avance en la capacidad de las baterías. Los dispositivos electrónicos usan relativamente poca potencia, por lo que incluso pequeñas mejoras en las baterías han impulsado grandes cambios en su desempeño y empleabilidad diaria. Es afortunado que la utilidad de las computadoras y teléfonos portátiles haya promovido este desarrollo, al punto que las baterías de hoy comienzan a ofrecer alternativas para incluso echar a andar automóviles.

El principio general de funcionamiento de cualquier batería tradicional (voltaica o galvánica) es el mismo: un ánodo y un cátodo, separados por un electrolito, que sufren reacciones de óxido-reducción que entregan y reciben electrones en un circuito. Los electrones viajan del ánodo al cátodo pasando de un estado de alta energía a otro de baja energía. En consecuencia, el voltaje de la batería está determinado físicamente por la diferencia de energía potencial que tienen los electrones cuando están asociados al ánodo (alta) y la que tienen cuando se asocian al cátodo (baja). Cuando el material del ánodo termina de oxidarse, o el cátodo termina de reducirse, la batería ha entregado ya toda la energía que tenía almacenada.

Desde mediados del siglo XX hubo interés en el litio como posible componente de un nuevo tipo de baterías ya que es el elemento que más fácilmente dona un electrón. Con esta motivación, por muchos años se fueron logrando avances respecto de cómo manejar este material que, aunque era muy prometedor, también era muy reactivo y había que protegerlo del agua y del aire. La minuciosa y larga investigación con materiales que intercalaban átomos y iones indujo, luego, a un radical cambio de ideas. Se observó que ciertos iones podían viajar en sustancias sólidas a gran velocidad, y que era además posible albergar estos iones de manera reversible. Así, M. Stanley Whittingham logró desarrollar

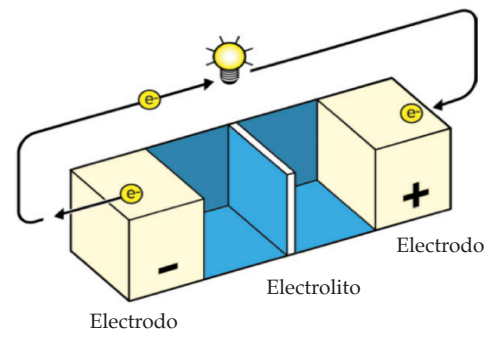

Por ejemplo:

$$
\begin{array}{cc}
\mathrm{Pb}+\mathrm{HSO}_{4}^{-} \rightarrow \mathrm{PbSO}_{4}+\mathrm{H}^{+}+2 e^{-} \mid & \mathrm{PbO}_{2}+3 \mathrm{H}^{+}+\mathrm{HSO}_{4}^{-}+2 e^{-} \rightarrow \mathrm{PbSO}_{4}+2 \mathrm{H}_{2} \mathrm{O} \\
\mathrm{Pb}^{0} \rightarrow \mathrm{Pb}^{2+}+2 e^{-} & \mathrm{Pb}^{4+}+2 e^{-} \rightarrow \mathrm{Pb}^{2+}
\end{array}
$$

Figura 1. Principio general de funcionamiento de una batería. El ánodo se oxida y entrega electrones al circuito. El cátodo se reduce tomando electrones del circuito. Como ejemplo se muestran las reacciones de una batería de plomo ácido, conocida por utilizarse en los automóviles comunes. En azul, los cambios de estados de oxidación del plomo de cada electrodo como una semirreacción simplificada.

Ilustración de uso libre (C) Johan Jarnestad/The Royal Swedish Academy of Sciences. 
una batería en la que el litio funcionaba como ánodo y los iones $\mathrm{Li}^{+1}$ viajaban como parte del electrolito hasta acomodarse en el cátodo (Figura 2). Además, introduciendo una fuente de energía externa, se podía inducir el camino inverso con altas tasas de transferencia, logrando así una batería recargable.

Lamentablemente, el ánodo de litio metálico resultó peligroso. Durante la fase de recarga, el ion $\mathrm{Li}+1$ que viaja de regreso a formarse como metal puede irse depositando en forma de largos 'bigotes'; estos pequeños cables, si llegan a alcanzar al cátodo, causan un cortocircuito (Figura 3). Los laboratorios que accidentalmente observaron estas fuertes descargas de energía sufrieron varios incendios.

La solución definitiva llegó gracias a los aportes de las investigaciones de John B. Goodenough y Akira Yoshino que permitieron desarrollar una batería en la que se prescinde totalmente del litio metálico. Así, tanto el ánodo como el cátodo están formados por materiales

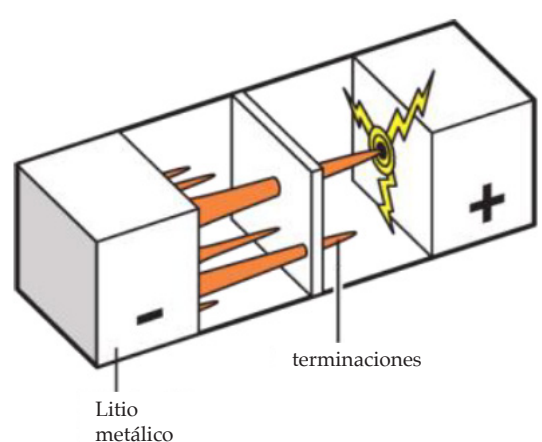

Figura 3. Un caso peligroso. El ánodo de litio metálico se deforma tras varios usos. Las prolongaciones de litio causan un cortocircuito y una descarga violenta de energía. Para lograr mayor seguridad, el desarrollo de las nuevas baterías abandonó el uso del litio metálico como ánodo.

Ilustración de uso libre (C) Johan Jarnestad/The Royal Swedish Academy of Sciences.

intercaladores, y el ion $\mathrm{Li}+1$ puede viajar de manera reversible entre ellos. Esta batería (Figura 4) utiliza grafito en el ánodo, que alberga átomos de litio de manera intercalada. Rodeado de grafito, el litio está protegido de reaccionar con aire o agua por tiempo prácticamente indefinido. Al conectarse, el átomo Li0 que es altamente inestable puede

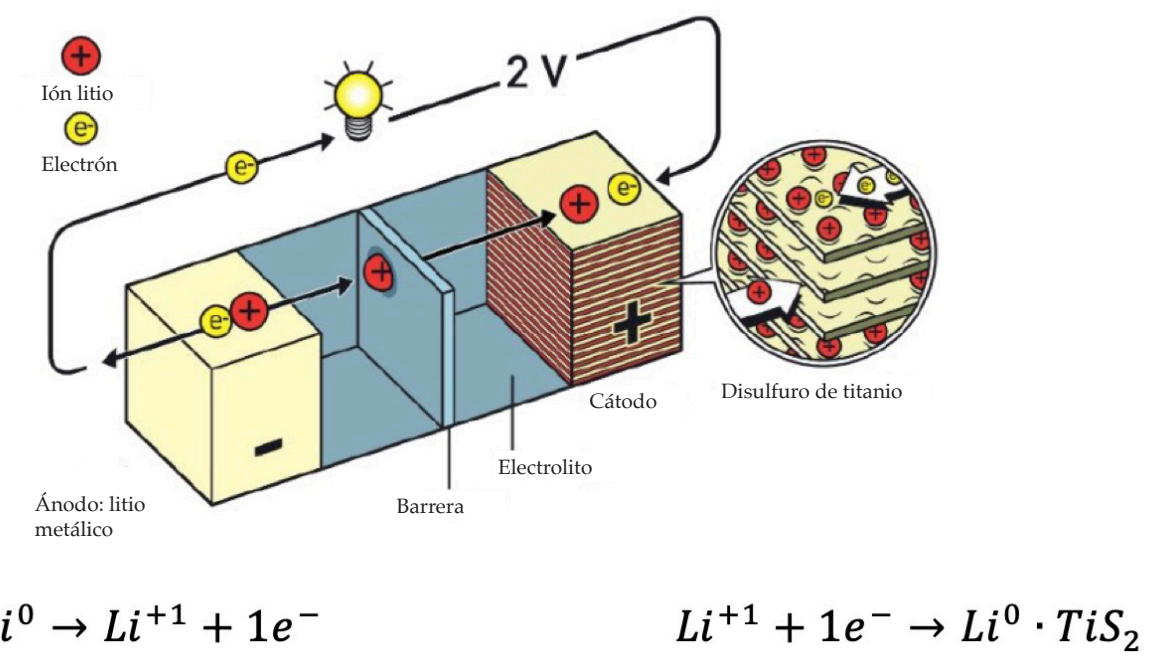

Figura 2. Primeras baterías de litio. El ánodo está construido de litio metálico. El Li0 entrega electrones al circuito y los iones $\mathrm{Li}+1$ resultantes viajan hacia el cátodo. El cátodo está constituido por disulfuro de titanio, un material con capacidad de recibir electrones y albergar iones $\mathrm{Li}+1$ intercalados. Ilustración de uso libre $@$ Johan Jarnestad/The Royal Swedish Academy of Sciences. 
entregar su electrón al circuito, se transforma en ion $\mathrm{Li}+1$ y puede abandonar el ánodo. La forma iónica $\mathrm{Li}+1$ viaja por difusión a través de una barrera sólida hasta llegar al cátodo. El cátodo es un óxido metálico que atrapa al átomo de litio de manera muy favorable: el ion Li+1 entra al material y recibe el electrón que 'cae' del circuito. El litio en su forma neutra Li0 es estable cuando está atrapado en este material. La batería puede recargarse (Figura 5) si se aplica una fuerte diferencia de potencial con una fuente de energía externa, así el electrón puede extraerse del óxido metálico y el ion $\mathrm{Li}+1$ hace el viaje reverso hasta llegar nuevamente al grafito donde recibe de vuelta su electrón.

En teoría, gracias al viaje reversible del ion $\mathrm{Li}+1$, se podría almacenar energía infinitas veces; sin embargo, en cada ciclo puede ocurrir pequeños accidentes que van acumulando imperfecciones en el material. Las baterías de este tipo actualmente utilizadas en los teléfonos móviles resisten cientos de ciclos de recarga. Las baterías más avanzadas en los autos Tesla resisten miles de ciclos de recarga. Se piensa que, al reemplazar el grafito por una estructura de silicio, se podrá fabricar baterías capaces de trabajar por cientos de miles de ciclos de recarga.

Una batería de litio puede almacenar 0,20,6 kWh de energía por litro. Por lo tanto, la gasolina, con $9 \mathrm{kWh} / 1$ todavía es un orden de magnitud más compacta que las mejores baterías de hoy. Pero, con un ritmo de mejora que aproximadamente duplica la densidad cada diez años, las baterías ya comienzan a ofrecer verdaderas alternativas para cada vez más escenarios. Los grandes capitales y especuladores ya prevén gigantescos mercados para las baterías que están por desarrollarse.

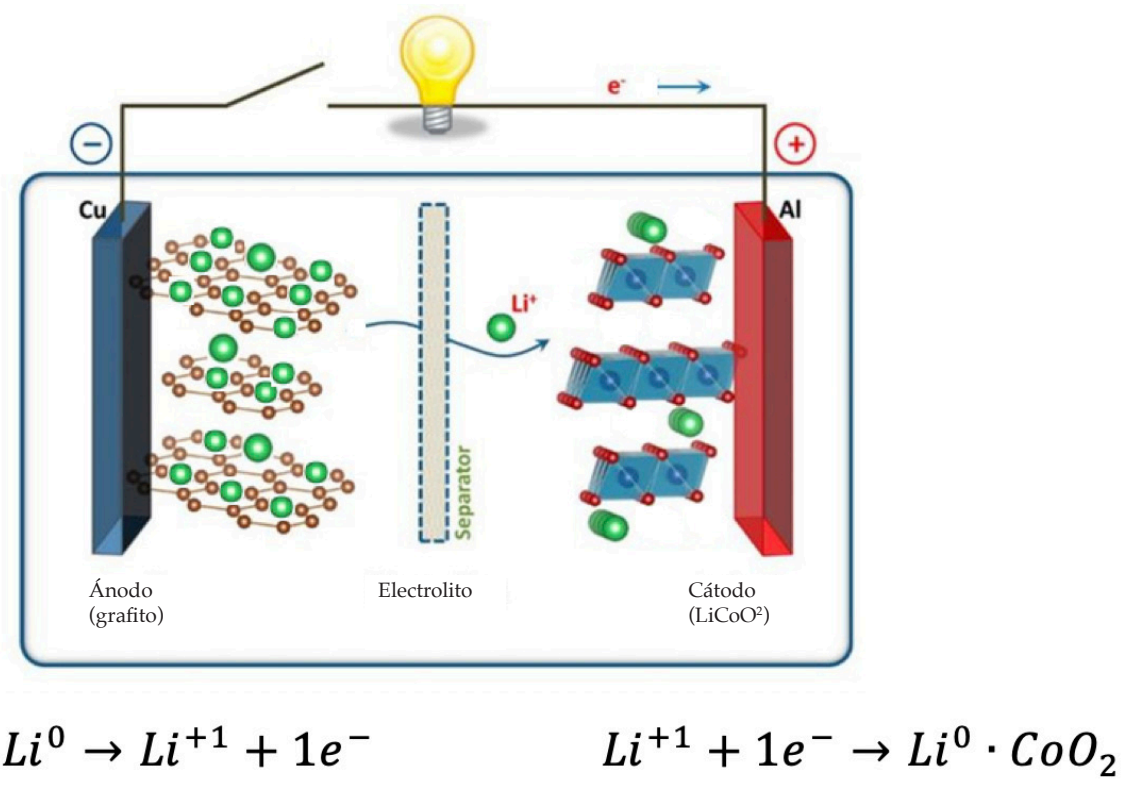

Figura 4. Batería de ion litio en fase de descarga. El átomo de litio albergado en el grafito entrega su electrón a una plancha de cobre conectada a un circuito que alimenta un trabajo útil, en este caso un foco de luz. El electrón viaja por el circuito hasta una plancha de aluminio en contacto con un óxido metálico de litio y cobalto. Por su parte, el ion $\mathrm{Li}+1$ abandona el grafito y atraviesa la batería hasta llegar al óxido metálico. En la matriz de $\mathrm{LiCoO} 2$, el ion $\mathrm{Li}+1$ recibe el electrón y es atrapado en forma estable como Li0.

Figura adaptada de Asghar et al., 2019. 


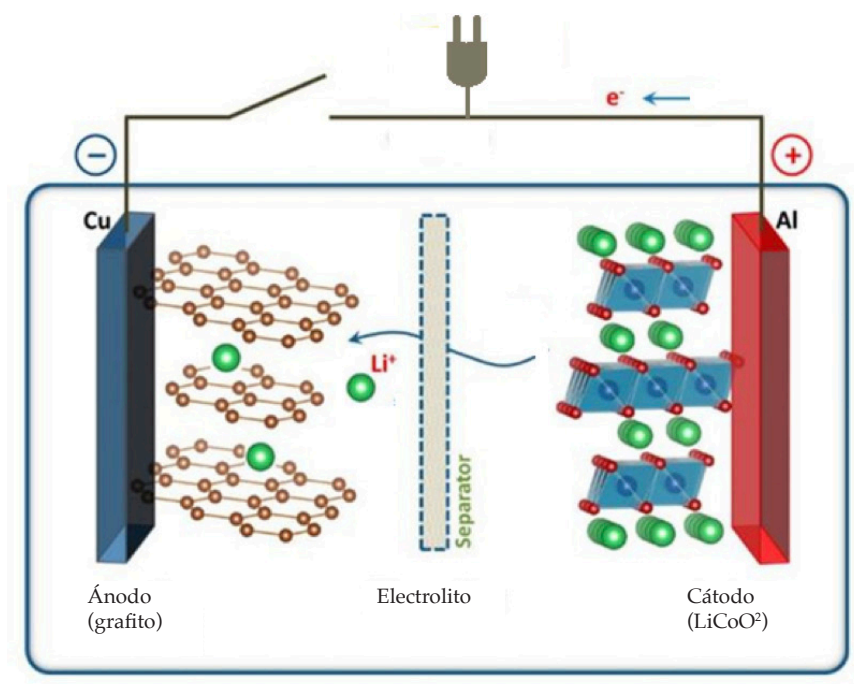

$$
L i^{0} \leftarrow L i^{+1}+1 e^{-} \quad L i^{+1}+1 e^{-} \leftarrow L i^{0} \cdot \mathrm{CoO}_{2}
$$

Figura 5. Batería de ion litio en fase de recarga. El átomo de litio atrapado en forma estable en el óxido metálico es ionizado por la acción de una fuente externa de energía, representado con un enchufe. El ión $\mathrm{Li}+1$ abandona el óxido metálico y viaja hasta llegar al grafito. En la matriz de grafito, enriquecida con electrones, el ion $\mathrm{Li}+1$ se reduce. Los átomos de $\mathrm{Li} 0$ capturados en el grafito son una forma química relativamente inestable, que queda lista para donar nuevamente sus electrones al circuito en el próximo ciclo de trabajo y descarga.

Figura adaptada de Asghar et al., 2019.

Podemos avizorar grandes cambios que se vienen en lo social, ambiental, económico y geopolítico para cuando las baterías alimenten la mayoría de los automóviles, fábricas y casas. En el sur de Australia, la famosa compañía Tesla, dirigida por Elon Musk, construyó la batería más grande del mundo en el año 2017, con la capacidad de almacenar 100 MWh. Con ella, se ha mejorado la eficiencia de la red que ya había fallado en suplir picos de alta demanda, causando apagones. En un futuro cercano, se espera que este tipo de baterías pueda incrementar la utilidad de la energía solar, almacenando energía en el día y proveyéndola a la red durante la noche. En el año 2019 se aprobó ampliar la gran batería en un 50\% para un total de 150 MWh (Harmsen et al, 2019).

Aunque las proyecciones de expansión del uso de baterías indican cada vez mayor demanda de litio y cobalto, la compleja cadena de explotación y manufactura genera oscilaciones que dejan confundido a los inversionistas. El precio del litio y cobalto subieron con locura solo para volver a caer el año 2019. ¿Cómo está cambiando la geopolítica mundial? Los yacimientos de cobalto en el Congo proveen el $54 \%$ de la producción mundial y son ya explotados por capitales chinos en una estrategia por controlar el futuro mercado de baterías de automóviles eléctricos. De hecho, China produce el $80 \%$ de sulfato de cobalto del mundo (Gold, 2018).

Queda por verse qué rol jugará el Perú en estos cambios. El acoplar grandes baterías a la red de suministro eléctrico será cada vez más importante para aumentar la eficiencia de uso de energía y evitar la pérdida de tensión. Por otro lado, tanto Perú como Bolivia han dado a conocer grandes yacimientos de litio 
recientemente. La demanda por este metal irá incrementando hasta que la inversión por su explotación en el Perú será una realidad. ¿Esta riqueza podrá contribuir al desarrollo de nuestro país, o será un caso más de industria extractiva que muy poco deja al territorio explotado?

\section{ReFERENCIAS BibLIOGRÁFICAS}

1. Asghar, M. R., Anwar, M. T., \& Naveed, A. (2019). A review on inorganic nanoparticles modified composite membranes for lithium-ion batteries: Recent progress and prospects. Membranes. 2019; 9(7):78-115. URL disponible en: https:// doi. org $/ 10.3390 /$ membranes 9070078

2. Gold R and Patterson S. There's a global race to control batteries - And China is winning. Wall Street Journal. February 18, 2018. URL disponible en: https://www.wsj.com/ articles / theres-a-global-race-to-control-batteriesand-china-iswinning-1518374815
3. Harmsen $\mathrm{N}$ and staff. Morrison likened it to the big banana, but South Australia's big battery is now getting big dollars. $A B C$ News. URL disponible en: https://www.abc.net.au/ news / 2019-11-19/sa-big-battery-set-to-get-evenbigger/ 11716784

4. Ramström O. Scientific background on the Nobel Prize in Chemistry 2019. Lithium-ion batteries. 2019, October 9.

5. Advanced information. NobelPrize.org. URL disponible en: https: / / www.nobelprize.org/uploads / 2019/10/ advanced -chemistryprize2019-2.pdf

\section{AGRADECIMIENTOS:}

A Vanessa Leyva Zegarra, por una revisión crítica del documento.

\section{CORRESPONDENCIA:}

Juvenal.Castromonte@upch.pe

FECHA DE RECEPCIÓN: 23-01-2020.

FECHA DE ACEPTACIÓN: 18-02-2020. 